\title{
COMPORTAMENTO, MANEJO E BEM-ESTAR DE MAMÍFEROS NEOTROPICAIS DE INTERESSE ZOOTÉCNICO
}

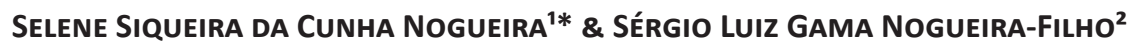

${ }^{1}$ Laboratório de Etologia Aplicada, Universidade Estadual de Santa Cruz, Ilhéus, Bahia, Brasil. *E-mail para correspondência: seleneuesc@gmail.com

${ }^{2}$ Laboratório de Nutrição de Animais Neotropicais, Universidade Estadual de Santa Cruz, Ilhéus, Bahia, Brasil. E-mail: slgnogue @uesc.br

\begin{abstract}
Behavior, management and welfare of Neotropical mammals with zootechnical interest. Farming and ranching of wild animals to sustainably meet increasing demands for animal protein in developing countries, albeit controversial, is a reality in several countries. Therefore, we need to develop production systems that promote the welfare of these animals. In this review paper, we described the challenges faced by Neotropical mammals maintained in captivity. We showed how the motivation absence or predictability in captive environment, such as the fixed time for feeding, affects the animals. Against these, we propose the use of some techniques to detect problems and to promote the welfare of Neotropical mammals. We also highlighted the importance of using knowledge of social behavior to achieve success in their production with less stress for the animals. We also showed that the behavioral plasticity of some species can be used to establish management practices that improve the feasibility of farming wild mammals without compromising their welfare. Moreover, we pointed out some gaps in our knowledge, especially in descriptions of normal/abnormal behavioral patterns. We concluded by proposing that some of the discussed techniques, such as the use of feeders that promote animal activity and the use of bioacoustics as stress indicator, should be improved and tested in other wild species maintained in captivity with different aims, such as zoos, conservation centers and, especially, on commercial
\end{abstract} farms.

Keywords: applied ethology, captive breeding, game ranching, stress, wildlife farming.

Resumo. A criação de animais não convencionais para atender a crescente demanda por proteína de origem animal de países em desenvolvimento, apesar de ser controversa, é uma realidade mundial. Desta forma, é preciso estabelecer sistemas de produção que promovam o bem-estar desses animais. Neste artigo de revisão, foram descritos alguns dos desafios que animais neotropicais enfrentam ao serem mantidos em cativeiro, tais como os efeitos da ausência de motivação e da previsibilidade no ambiente onde são criados, como horários fixos de alimentação, que comprometem seu bem-estar. Para contrapor, foram mostradas algumas técnicas para detectar problemas e para promover o bem-estar de mamíferos neotropicais com potencial zootécnico. Também foi reforçada a importância do uso de conhecimentos sobre o comportamento social para alcançar o sucesso na sua produção respeitando suas características e necessidades comportamentais. Por outro lado, foi mostrado que a plasticidade comportamental, exibida por algumas espécies, pode ser aproveitada para estabelecer práticas de manejo que viabilizem a atividade, sem comprometer seu bem-estar. Adicionalmente, foram apontadas as lacunas em nosso conhecimento, especialmente os relativos aos comportamentos típicos e/ou anormais. Conclui-se que as técnicas discutidas, como as de manejo alimentar que estimulam a atividade dos 
animais e de monitoramento do estresse por meio da bioacústica, aqui apresentadas e discutidas, sejam aperfeiçoadas e testadas na produção das diversas espécies de animais neotropicais mantidas em cativeiro, mesmo que estejam em cativeiro com diferentes objetivos, tais como em zoológicos, centros de triagem e reabilitação, centros de multiplicação de animais destinados a programa de soltura e, especialmente, nas criações comerciais.

Palavras-chave: animais silvestres, criação em cativeiro, estresse, etologia aplicada.

\section{INTRODUÇÃO}

A criação de animais não convencionais para atender à crescente demanda por proteína de origem animal da população humana de países em desenvolvimento, apesar de ser controversa (HOFFMAN, 2016), é uma realidade mundial, tanto no sistema confinado ou semiconfinado - wildlife farming (VAN VLIET et al., 2015) quanto em sistemas mais extensivos game ranching (PITMAN et al., 2017). Na América do Sul, encontramos um dos exemplos de maior sucesso no uso de animais não convencionais o porquinho-da-índia (Cavia porcellus, Erxleben, 1777). Esta espécie foi domesticada no período pré-colombiano - em torno de 3000 a 6000 anos atrás na região andina (STAHL, 2003). Apesar disso, apenas nas últimas décadas do século $X X$ que pesquisas mais intensas foram realizadas e promoveram a seleção de animais mais calmos, precoces e prolíficos (CHAUCA, 1997). A criação dessa espécie, que se adapta a diferentes condições climáticas e tipos de dieta, garante a segurança alimentar de milhares de pessoas (CAWTHORN \& HoffMAN, 2016). No Peru são abatidos anualmente 64 milhões de porquinhos-da-índia, produzindo em torno de 20 mil toneladas de carne (KYLE, 1994), e no Congo, ao menos dois milhões de porquinhos-da-índia são criados para o consumo humano (METRE et al., 2014). Na América do Sul, outras espécies como capivara (Hydrochoerus hydrochaeris, Linnaeus, 1766) e caititu (Pecari tajacu, Linnaeus, 1758), foram extensivamente estudadas, desde o texto seminal de Nogueira Neto (1973), o que possibilitou o desenvolvimento de técnicas para sua produção em cativeiro, que mostram a viabilidade e o potencial econômico da atividade (Nogueira-Filho \& Nogueira, 2004; NogUeiraFILHo et al., 2013). Apesar disso, a produção no Brasil dessas e outras espécies autóctones continua a ser considerada como incipiente (LE Pendu et al., 2011). Este cenário é decorrente de vários fatores tais como falta de incentivo governamental, falta de mão de obra técnica especializada, número pequeno de pesquisadores dedicados aos temas como melhoramento genético, sanidade, nutrição, comportamento e bem-estar de animais neotropicais (NogUeIRAFILHO \& NogUeIRA, 2004; NogUeIRA \& NogUeIRA FILHO, 2011).

O principal gargalo enfrentado pelos produtores rurais, no entanto, é o arcabouço legal extremamente burocrático, tanto para implantação dos criadouros (NOGUEIRA-FILHO \& NogueIRA, 2004), quanto para comercialização dos produtos oriundos de animais nativos, 
mesmo os provenientes de criadouros legalmente registrados (VAN VLIET et al. 2016). Por esses motivos, produtores interessados na atividade são desestimulados a iniciar ou ampliar suas criações. No Brasil, há um número relativamente reduzido de criadouros de animais neotropicais registrados junto ao Instituto Brasileiro de Meio Ambiente e Recursos Renováveis (IBAMA): 194 são criadouros de mamíferos, 230 criam aves e apenas 163 criam répteis (LE PENDU et al., 2011). A complexidade na legislação decorre, em grande parte, devido à falsa percepção, tanto por parte dos agentes responsáveis pela elaboração e fiscalização dessa legislação quanto por grande parte da sociedade que vive em regiões urbanas, de que animais da fauna nativa seriam 'sagrados' e, por isso, devem apenas ser protegidos.

$\mathrm{Na}$ realidade, a carne de animais autóctones ainda representa grande parte da proteína de origem animal consumida por populações tradicionais e comunidades rurais com condições socioeconômicas menos privilegiadas, como indígenas, seringueiros, quilombolas e caiçaras, que habitam florestas em diversas regiões do Brasil, tanto em áreas de floresta da Amazônia e Mata Atlântica, quanto no semiárido nordestino (MONEGo et al., 2010; ISAAC et al., 2015; VAN VLIET et al., 2015 BARBOzA et al., 2016; SiLVA Neto et al., 2016). Apesar disso, a caça de subsistência é ignorada em nossa legislação e é responsável pelo fenômeno da 'floresta vazia', descrito por REDFORD (1992). Esse autor mostrou que a perseguição persistente de algumas espécies ameaça a manutenção de sistemas florestais. Por exemplo, o caititu que é um dos animais mais caçados na região amazônica (BODMER et al., 2004), tem uma ação fundamental na predação/dispersão de sementes (BECK, 2005). Essa espécie, no entanto, devido a sua ampla distribuição e capacidade de adaptação a ambientes alterados pela ação humana, é considerada pela União Internacional para a Conservação da Natureza (IUCN) como 'menos preocupante' (least concern, GoNGORA et al., 2011). Em algumas regiões do Brasil, os produtores rurais consideram o caititu como 'praga agrícola' (NogueIRA-FILHO \& NogueIRA, 2004). O mesmo ocorre com a capivara (ABREU Bovo et al., 2016) e a paca (Cuniculus paca, Linnaeus, 1766) (LobÃo \& NogueIRA-FIlHo, 2011), que são perseguidas devido aos prejuízos que provocam em cultivos agrícolas, como cana de açúcar, milho e cacau.

Por esses motivos, consideramos que a produção de animais neotropicais pode ser considerada como parte da resolução do conflito Homem $X$ Fauna silvestre, transformando 'animais praga' em fonte de renda para os produtores rurais (NogUEIRA-FILHo et al., 2013). Por outro lado, a criação desses animais pode garantir a segurança alimentar de populações que vivem em regiões onde as condições locais, tais como temperatura, umidade e cobertura vegetal, limitam o potencial de produção de espécies criadas tradicionalmente - todas exóticas (NogueIRA-Filho \& NogueIRA, 2004; Nogueira \& NogUeIRA-FILHO, 2011). Em sistema agroflorestal, com o uso de coprodutos agrícolas 
disponíveis localmente para sua alimentação, a produtividade de caititus é de $700 \mathrm{~kg}$ de carne/ hectare/ano (NogueIRA \& NogueIRA-Filho, 2011). Esse valor é 19.000 vezes maior do que a produtividade da espécie alcançada por meio do manejo sustentável (36 g de carne/hectare/ ano, BODMER \& RoBINSON, 2004). Desta forma, a criação de caititu, capivara e paca atendem aos pré-requisitos destacados por BULTE \& DAMANIA (2005), que recomendam apenas a criação de animais que apresentem demanda e que não estejam ameaçadas.

Dado este cenário, no presente artigo, tivemos como objetivo principal destacar os estudos sobre o comportamento e fisiologia que têm mostrado beneficiar 0 bem-estar dessas espécies em sistema de cativeiro, além de avanços alcançados no manejo de mamíferos neotropicais, tanto para produção comercial quanto para programas de conservação - como reintrodução em áreas onde foram extintos. Também procuramos apontar as lacunas a serem preenchidas com novas pesquisas que promovam o bem-estar dessas espécies, e consequentemente sua produtividade. Esperamos, portanto, que este artigo sirva como base para novos estudos que proporcionem a ampliação do conhecimento sobre as espécies neotropicais mantidas em cativeiro.

\section{Os Desafios do Ambiente de Cativeiro}

Ao longo do processo evolutivo, as espécies neotropicais adaptaram-se a ambientes complexos, tais como florestas densas com grandes corpos d'água e ambientes amplos da savana e caatinga. Nesses locais, os animais forrageiam, buscam abrigo e se protegem de predadores. O ambiente de cativeiro, no entanto, traz uma nova perspectiva para esses animais, alterando seu programa comportamental, fazendo com que tenham de se adaptar a uma nova realidade em que há perdas e ganhos no que diz respeito ao seu bem-estar. Um dos benefícios do cativeiro para os animais é o recebimento de alimento, sem a necessidade de busca. Os horários determinados para a alimentação, bem como para as demais atividades rotineiras, como limpeza das instalações, podem, no entanto, proporcionar uma previsibilidade ambiental muitas vezes negativa (WAITT \& BUCHANAN-SMITH, 2001; WATERS, 2009). A previsibilidade apresenta aspectos tanto negativos quanto positivos, que devem ser considerados no que diz respeito ao bem-estar de animais em confinamento. Por um lado, ocorre a falta de motivação causada pela ausência de estímulos novos e inativadade devido à segurança das necessidades atendidas. Com a falta de estímulos novos e de um ambiente que não gera motivação, esta condição pode favorecer o desenvolvimento de comportamentos anormais como estereotipias (MASON et al. 2007), a apatia ou inatividade (WATERS, 2009). Por outro lado, a previsibilidade pode trazer segurança ao animal, por diminuir sua ansiedade, que resulta em distresse (estresse negativo) devido à escassez de alimento ou quando há receio de ser predado durante forrageamento/alimentação. 
Considerando esses aspectos sobre a previsibilidade ambiental, foram avaliados os efeitos de um ambiente imprevisível usando as técnicas de enriquecimento ambiental (EA) para a alimentação de caititus (NogueIRA et al. 2011a) e queixadas (Tayassu pecari, Link, 1795) (Nogueira et al., 2014) mantidos em cativeiro. Esses estudos foram feitos porque, de modo geral, nos criadouros comerciais de caititus e zoológicos (Nogueira-Filho, observação pessoal), os animais são alimentados em cochos uma única vez ao dia, com ração formulada para porcos domésticos, que pode ser suplementada com mandioca e frutas em horário fixo e conveniente ao proprietário/tratador. Para promover imprevisibilidade ambiental, foi aplicado no estudo o esquema $A_{1} B_{1} A_{2} B_{2}$ proposto por HEFFNER (2004), em que cada uma das fases $A$ (controle) e fases $B$ (tratamento) tiveram a duração de 15 dias. As fases controle $\left(A_{1}\right.$ e $\left.A_{2}\right)$ corresponderam à rotina do criadouro de fornecimento do alimento (local e horários fixos). Durante as fases B foram testados dois tratamentos de enriquecimento alimentar. Na fase $B_{1}$, foi testada a imprevisibilidade espacial do local da oferta do alimento, enquanto que na fase $B_{2}$ testou-se a imprevisibilidade temporal da oferta do alimento. Para isso, foram construídos comedouros desafio, feitos com tubo de PVC $(1,0 \mathrm{~m}$ de altura e $150 \mathrm{~mm}$ de diâmetro), com uma porta retrátil $(0,30 \mathrm{~m}$ altura vs. 0,15m comprimento) em sua base. Desta forma, para acessar o alimento, os animais tinham um certo trabalho para abrir e manter aberta a porta do comedouro que estava ligada à parede do mesmo por uma mola que forçava seu fechamento. Durante a fase de imprevisibilidade espacial $\left(B_{1}\right)$, o alimento foi fornecido em dois horários fixos (as 08h:00min da manhã e as 5h:00min da tarde), mas do total de quatro comedouros disponíveis aos animais, apenas dois (proporção de três animais por comedouro) foram preenchidos com o alimento rotineiro, seguindo um sorteio prévio de comedouros a serem preenchidos. Esta prática forçava os animais a forragearem entre os comedouros para descobrir qual continha o alimento. Por sua vez, na fase de imprevisibilidade temporal $\left(B_{2}\right)$, a alimentação foi fornecida em todos os comedouros, mas em dois horários sorteados ao longo do dia. Deste modo, os animais não sabiam quando receberiam a alimentação. Para ambas as fases tratamento $\left(B_{1}\right.$ e $\left.B_{2}\right)$, os caititus reduziram o tempo de inatividade em $37 \%$, aumentaram em $48 \%$ o comportamento exploratório e triplicaram o tempo gasto na alimentação em relação às fases controle $\left(A_{1} e\right.$ $A_{2}$ ), em que seguiram a rotina de previsibilidade alimentar. Adicionalmente, houve diminuição dos comportamentos agonísticos durante os períodos de enriquecimento ambiental. Desta forma, com ambos os tipos de imprevisibilidade (temporal e espacial), foi possível aumentar comportamentos considerados positivos do ponto de vista de bemestar para caititus (comportamento exploratório e alimentar), revelando que a imprevisibilidade beneficia a espécie, aumentando seu tempo de forrageio em detrimento da inatividade.

Devido aos resultados positivos para caititus, também foram testados os efeitos da 
imprevisibilidade em queixadas, também com objetivo de estimular a atividade dos animais. Esta espécie está classificada como vulnerável pela IUCN (KeURoghliAN et al., 2013) em razão de sua extinção em vários locais de distribuição natural. Queixadas em vida livre percorrem em média 10 $\mathrm{km}$ por dia em atividade de forrageio (FRAGoso, 1998), assim a rotina e espaço limitados do ambiente de cativeiro podem afetar habilidades naturais da espécie para a busca de alimento, tornando-os mais sedentários. Provavelmente, este fato explique o fracasso na reintrodução de um grupo de queixadas nascidos e criados em cativeiro, que após sua soltura em uma reserva florestal, ficaram dependentes da alimentação fornecida pelos pesquisadores (FIGUEIRA et al., 2003). Desta forma, pretendemos verificar se animais nascidos e criados em cativeiro passariam a gastar mais tempo em atividades de forrageio, com a finalidade de potencializar as habilidades necessárias para sua soltura na natureza em programas de reintrodução (NogUEIRA et al., 2014).

É senso comum que, para se obter sucesso em programas de soltura de animais oriundos de cativeiro, é fundamental habilitá-los ou reabilitálos para sua sobrevivência na natureza, além de selecionar indivíduos saudáveis do ponto de vista físico e psíquico e bem aparelhados em habilidades locomotoras. Deste modo, ao estabelecer um programa de treinamento présoltura para queixadas, foi testado o uso de EA associado à imprevisibilidade ambiental visando aumentar seu comportamento exploratório
(Nogueira et al., 2014). Neste programa foi seguido o protocolo experimental de HEFFNER (2004), similar ao descrito anteriormente para caititus $\left(A_{1} B_{1} A_{2} B_{2} A_{3}\right)$, exceto pelo acréscimo de uma fase controle final $A_{3}$ para avaliar o efeito posterior ao EA em $B_{1}$ e $B_{2}$. Além disso, durante a fase de imprevisibilidade temporal $\left(B_{1}\right)$ também foi incluído um sinal seguro (sinal de apito) para indicar a oferta do alimento. Antes de iniciar o estudo, os animais foram treinados, por meio de condicionamento clássico, a ir até o local de alimentação após o acionamento do apito. $O$ sinal seguro foi liberado sempre que o alimento foi fornecido, independente do horário, com intuito de diminuir a ansiedade dos animais seguindo a hipótese de WATERS (2009).

Os resultados mostraram que, nas duas fases de tratamento com EA, houve aumento do comportamento exploratório de queixadas em relação às fases controle. Adicionalmente, verificou-se o efeito de aumento do tempo gasto no comportamento exploratório arrastando-se para a fase controle $\left(A_{2}\right)$ que ocorreu após a fase $B_{1}$, revelando que a segurança da perspectiva da presença do alimento dada pelo sinal, torna os animais exploradores por mais tempo (NogUEIRA et al., 2014). A partir desses resultados, podemos sugerir que em programas de reintrodução sejam usados dispensadores automáticos, que sejam programados para liberar o alimento em momentos aleatórios, ao mesmo tempo em que emitam um sinal acústico para alertar essa oferta. Este resultado, que deve ser testado para outras espécies, mostra que a 
imprevisibilidade temporal e espacial estimula a execução de comportamentos importantes para a sobrevivência de queixadas na natureza. Mais importante, mostrou-se que a imprevisibilidade temporal associada à emissão de um sinal seguro, resultou em um efeito duradouro na manutenção da atividade exploratória, o que será extremamente vantajoso após sua soltura na natureza onde precisam se manter em movimento para a busca de alimento e evitar predadores. Adicionalmente, este tipo de manejo também pode ser aplicado nas criações comerciais de queixadas, para mantêlos motivados e em boa forma.

O ambiente de cativeiro expõe os animais a fontes persistentes de estresse, tais como espaço reduzido, ausência de áreas de refúgio, além de outras restrições de oportunidades para o desenvolvimento dos comportamentos naturais da espécie, que podem acarretar em consequências deletérias ao seu bemestar (Morgan \& TROMborg, 2007; MCPheE \& CARLSTEAD, 2012). Portanto, outro aspecto fundamental para a manutenção adequada de mamíferos neotropicais em cativeiro é a avaliação do espaço disponível em relação ao número de animais. Devido a inexistência de estudo sistemático sobre esta relação entre disponibilidade de área e bem-estar de caititus, NogueIRA et al. (2010) avaliaram o efeito de três tamanhos de áreas $\left(375,750\right.$ e $\left.1.500 \mathrm{~m}^{2}\right)$ para criação de grupos de caititus, com oito indivíduos cada. Usando o delineamento quadrado latino (3 $X 3$ ), no qual cada grupo passou pelas três áreas testadas em três fases distintas, NogueIRA et al. (2010) avaliaram os efeitos do espaço sobre os comportamentos amigáveis e agressivos dos animais antes e durante a alimentação. Além disso, os autores testaram a necessidade do fornecimento de áreas de abrigo para esses animais, uma vez que na natureza, há relatos de que esses animais fazem o uso de buracos no solo e na base de troncos de árvores para se abrigarem (SowLs, 1997). Para este fim, foram introduzidos três abrigos em cada recinto, aproximadamente um abrigo para cada três animais. Verificou-se que houve menor frequência de comportamentos agonísticos durante a alimentação quando os caititus estavam no piquete com maior espaço disponível. Neste estudo, os autores também observaram que ocorreu maior uso dos abrigos à medida que o espaço disponível diminuía, sugerindo que esses abrigos também eram uma forma de proteção nos momentos de conflito. Os resultados mostraram que a melhor relação espaço/animal é em torno de $200 \mathrm{~m}^{2} /$ animal para criação da espécie, o que permite criar até 50 caititus por hectare no sistema de semiconfinamento. Também mostrou a importância da disponibilidade de abrigos para promover maior controle em situações de conflito (Nogueira et al. 2010). Por sua vez, AndRAde et al. (2009), comparando caititus criados em sistema confinamento intensivo, em baias com 3 a $16 \mathrm{~m}^{2}$, e semi-confinado, em piquetes de 1,0 hectare, verificaram que nos piquetes de semi-confinamento os caititus permaneceram mais tempo ativos, forrageando mais do que os mantidos no sistema de confinamento intensivo, 
no qual os animais passaram a maior parte do tempo inativos deitados ou parados em pé. Esses resultados indicam, portanto, que condições de bem-estar empobrecido do sistema de confinamento intensivo, por sua vez, afetaram a produtividade. No sistema confinado, os jovens apresentaram ganho de peso diário médio de $23,1 \pm 12,4 \mathrm{~g} / \mathrm{dia}$, inferior ao do semi-confinado, com média de 58,8 $\pm 12,2 \mathrm{~g} /$ dia (ANDRADE et al., 2009). As taxas reprodutivas também diferiram de acordo com o sistema de produção, enquanto no sistema confinado as fêmeas apresentaram média de dois partos por ano, com $1,5 \pm 0,7$ filhotes por parto e taxa de mortalidade de 33\%, no sistema semi-confinado as fêmeas apresentaram

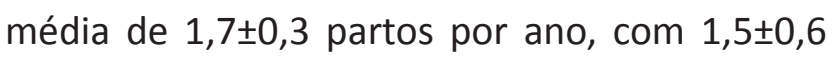
filhotes por parto e taxa de mortalidade de $8,3 \%$ (ANDRADE et al., 2009).

\section{ASPECtos dA VidA EM GRUPO E O BEM-ESTAR}

A vida em grupo e a existência de hierarquia de dominância estão entre as características que favoreceram a domesticação da maior parte das espécies tradicionalmente criadas atualmente (ZEDER, 2012). Por isso, ao incorporar novas espécies ao processo produtivo, devemos considerar e respeitar suas relações sociais para que não haja prejuízos quanto ao bem-estar psíquico e físico dos indivíduos, que certamente afetará a viabilidade da criação daquela espécie. A capivara, por exemplo, possui particularidades de sua organização social que podem beneficiar a sua criação. O não respeito a alguns aspectos básicos de sua sociabilidade, porém, podem levar ao fracasso da prática
(Nogueira et al., 1999; Nogueira \& NogueiraFILHO, 2012). Em vida livre, a espécie vive em haréns, com um macho dominando as fêmeas, que com seus filhotes são encontradas no centro do grupo, e outros machos subordinados mais na periferia (HERRERA, 2013).

A hierarquia de dominância linear caracteriza a estrutura social dos machos de capivaras em vida livre (HERRERA, 2013). Em cativeiro, no entanto, é possível manter apenas um macho no recinto, do contrário, o macho subordinado será constantemente perseguido pelo dominante e se tornará submisso até mesmo às fêmeas (NogUEIRA-FILHo et al., 2017). Em cativeiro, onde os recursos alimentares são restritos aos locais de fornecimento de alimento, as fêmeas podem apresentar uma hierarquia de dominância similar ao despotismo, com uma única fêmea dominando o comedouro e dificultando o acesso ao alimento as demais, como descrito por Ferraz et al. (2013). Caso o alimento seja espalhado em diversos locais, como recomendado por MENDES \& NOGUEIRA-FILHO (2013), elas exibem hierarquia de dominância linear, com tendência das mais pesadas ocuparem os postos de dominância (NogUEIRA-FILHo et al., 2017). Portanto, é importante que os produtores forneçam o alimento em vários locais, em geral feixes de 5,0 kg capim elefante (Pennisetum purpureum, Schum) dependurados em árvores (Mendes \& NogueirA-Filho, 2013), para evitar o monopólio de uma única fêmea ao alimento. Adicionalmente, a hierarquia de dominância linear é vantajosa porque, após 
estabelecida, as disputas limitam-se a confrontos simbólicos reduzindo a ocorrência de confrontos que envolvam contato físico.

Outra característica do comportamento social de capivaras que facilita sua criação é o fato das fêmeas apresentarem o comportamento creche - uma fêmea 'cuida' dos filhotes dela e das demais fêmeas do grupo, com a presença de amamentação cruzada - quando uma fêmea amamenta seus filhotes e das demais do grupo (Nogueira et al., 2000). Isso é possível porque as fêmeas de capivaras sincronizam o estro e, portanto, vários filhotes nascem com poucos dias ou semanas de diferença. Esta prática é vantajosa para o grupo, pois na falta da mãe biológica, outra fêmea do grupo pode amamentar seus filhotes. Esta informação é valiosa para a produção em cativeiro, uma vez que pode ser realizada a adoção de filhotes que perderam sua mãe no parto, por exemplo. Isso somente ocorre, porém, caso as fêmeas que são mantidas no mesmo recinto sejam familiarizadas entre si, caso contrário, podem morder e matar os filhotes umas das outras. De fato, o infanticídio pode representar até $40 \%$ das causas da mortalidade de filhotes de capivaras (NogueIRA et al., 1999; Nogueira \& Nogueira-Filho, 2012) e, por este motivo, deve-se manter no mesmo recinto apenas fêmeas familiarizadas - fêmeas que vivam junto ao menos desde o desmame aos 60 dias de vida. Do contrário, o produtor terá que isolar as fêmeas para parição aumentando seus custos de produção, com a construção de novas instalações e gasto com mão de obra (NOGUEIRA-FILHo et al.,
2013). Adicionalmente, ao evitar o isolamento das fêmeas para parição, por manter na criação apenas fêmeas familiarizadas, o produtor poderá beneficiar-se dos estros que ocorrem durante a amamentação, aumentando a produtividade da espécie (Nogueira-Filho \& NogueirA, 2013).

Caso o produtor queira introduzir filhotes órfãos de outros grupos, será necessário aplicar a técnica de mascaramento do odor, dessa forma aumentará a chance dos filhotes adotivos serem aceitos no novo grupo. Para esta prática, deve-se retirar todos os filhotes do grupo que receberá o(s) filhote(s) órfão(s). Em seguida, deve-se juntá-los - os 'residentes' e o(s) órfão(s) - em um recinto de manejo aonde serão pulverizados com uma solução de substância com odor forte, com o de cresóis conhecidos popularmente por creolina, que irá mascarar o odor original dos filhotes ('residentes' e 'órfãos'). Após esta pulverização todos os filhotes ('residentes' e 'órfãos') poderão ser soltos no piquete com os demais animais adultos. Esta prática mostrouse eficaz e permitiu a sobrevivência de ninhadas de capivaras deixadas na Universidade Estadual de Santa Cruz-UESC por agentes do IBAMA, que relataram a morte da mãe biológica da ninhada por caçadores.

As capivaras são extremamente territoriais, tanto em vida livre (HERRERA, 2013) quanto em cativeiro (NogueIRA \& NogueIRAFILHO, 2012); intrusos não são tolerados e, quando indivíduos de grupos distintos se encontram, os conflitos podem ser letais. Deste modo, verifica-se que as capivaras de alguma 
forma reconhecem os membros de seu grupo. Diferenças na comunicação vocal corroboram essa hipótese, pois estudos mostram que ocorre o reconhecimento entre grupos (BARROS et al., 2011), como também entre indivíduos de grupos distintos (LACERDA et al. 2013). Portanto, além das informações químicas relacionadas às glândulas de odor nas capivaras (MACDONALD et al., 1984), há transferência de informação vocal sobre as diferenças entre os grupos.

Caititus e queixadas vivem em grupos mistose estáveis na natureza, com adultos, filhotes e jovens de ambos os sexos (SowLS, 1997). Estas características facilitam, e muito, sua produção em cativeiro (NogUeIRA-FILHo, 1999), uma vez que as fêmeas podem parir no grupo e 15 a 20 dias após o parto poderão ser fecundadas, o que reduz o período entre os partos. Apesar de ser possível introduzir animais de grupos diferentes em colônias estabelecidas em ambas as espécies, essa pratica não é recomendada. Ao observarem grupos de caititus e queixadas nos quais foram introduzidos animais de procedências diferentes, NogUEIRA-FILHo et al. (1999) verificaram que, mesmo dois anos após a introdução, os animais ainda agiam de forma distinta de acordo com sua procedência. A maior parte das interações amigáveis foi dirigida aos animais de mesma procedência, enquanto que as agressões ocorreram, em sua maior parte, entre indivíduos de procedências diferentes. Ao mesmo tempo, porém, observaram o acasalamento entre machos e fêmeas de procedências distintas. Desta forma, os autores sugerem que sejam formados grupos apenas com fêmeas de mesma procedência ou familiarizadas e, para reduzir os efeitos da endogamia, recomendam que os produtores introduzam machos de grupos distintos. Por meio desse processo, alguns produtores conseguem formar grupos com mais de 400 caititus (NogueIRA-FILHo et al., 2004).

Algumascaracterísticas comportamentais, a princípio, desestimulam a criação de determinadas espécies. A paca, por exemplo, geralmente é descrita como um animal de hábito solitário e noturno na natureza (BECK-KING et al., 1999). Podemos, contudo, nos aproveitar do fato de que, em geral, as espécies apresentam plasticidade no seu comportamento. Existem relatos que, em condições de menor pressão de caça, as pacas formam grupos em vida livre (FigueRoA-DE-LeON et al. 2016). Em razão disso, SMYTHE (1991) estabeleceu um método para habituar filhotes de paca a viverem em grupo e a alterar seu ciclo cronobiológico de noturno para diurno. Adotando esse método, ao invés de casais de pacas, os produtores passaram a criar um macho e até cinco fêmeas em baias de 8-12 $\mathrm{m}^{2}$ e que se alimentam durante o dia. Alguns produtores conseguem formar grupos maiores com três a quatro machos e até 15 fêmeas em recintos com $30 \mathrm{~m}^{2}$ (NogUEIRA-FILHO, observação pessoal). Caso esses grupos sejam formados por animais que convivam juntos desde o desmame, não haverá necessidade de isolamento das fêmeas para parição e, com isso, o produtor reduzirá o intervalo entre partos devido à possibilidade de acasalamento durante o estro 
pós-parto (NogueirA-Filho \& NogueirA, 1999). A manipulação cronobiológica, no entanto, ainda necessita ser analisada com relação aos efeitos sobre o bem-estar dos animais.

Adicionalmente, estudos mostraram que um comportamento natural exibido pela paca, a cecotrofia - a ingestão do conteúdo de ceco rico em proteína microbiana - deve ser estimulado (SABATINI \& PARANHOS DA COSTA, 2001). A realização deste ato permite com que a espécie tenha baixa exigência proteica (NogueIRA-Filho et al., 2016). A paca apresenta níveis relativamente elevados de digestibilidade da porção fibrosa dos alimentos, o que possibilita incluir em sua dieta coprodutos agrícolas, tais como resíduos da industrialização de polpas de frutos e raspa de mandioca, o que reduz os gastos com sua alimentação (MoNTEIRO, 2014; MATTOS, 2015). Esse conjunto de fatores - socialização, aumento do número de partos por ano, habito diurno, redução nos custos com alimentação viabilizam economicamente a criação da espécie sem afetar o seu bem-estar, uma vez que são aproveitadas características comportamentais e fisiológicas naturais da espécie.

\section{INDICADORES DE BEM-ESTAR EM ANIMAIS NEOTROPICAIS}

Apresença de distúrbioscomportamentais indica que os animais estão em algum processo de sofrimento ou comprometimento de seu bem-estar (HOSEY et al., 2009). A prevenção do aparecimento de tais distúrbios tem sido apontada como o melhor caminho para manutenção saudável de animais silvestres em cativeiro (HOSEY et al., 2009). Como pouco se sabe sobre o comportamento natural da maioria das espécies silvestres, há necessidade de indicadores ou métodos que revelem falência no sistema psíquico ou fisiológico desses animais, apontando distúrbios comportamentais e empobrecimento de bem-estar, para que se possa implantar melhorias necessárias o mais rapidamente possível. Neste contexto, nos últimos anos tem-se trabalhado com indicadores comportamentais, fisiológicos e cognitivos. Sabe-se, por exemplo, que o comportamento de brincadeira (FAGEN, 1981) é praticado quando os animais estão em boa condição física e psíquica (Bekoff \& BYERS, 1998). Portanto, a expressão deste tipo de comportamento tem sido relacionada como um indicador de bemestar animal (HELD \& SPINKA 2011; NogueIRA et al. 2011b).

Em geral, a brincadeira é mais observada em animais jovens (BEKOFF \& BYERS, 1998). Queixadas, entretanto, são animais que expressam o comportamento de brincadeira também na idade adulta e, por isso, NogueIRA et al. (2011b) testaram os efeitos do enriquecimento ambiental (EA) sobre o comportamento de brincadeira na espécie. Neste estudo, foi avaliado o uso de três objetos novos - uma bola, uma gangorra de bambu e uma mangueira como EA físico no recinto de 24 queixadas (seis machos, seis fêmeas, nove jovens e três filhotes). Esses objetos foram colocados no recinto, um de cada vez, para avaliar seu efeito individual 
sobre a brincadeira do grupo social, seguindo um esquema ABA (Fases A: controle sem EA; Fase B: fase experimental com EA). No estudo, ainda foi avaliado o efeito do enriquecimento ambiental sobre comportamentos agonísticos do grupo e inatividade, como também a relação entre brincadeira com a hierarquia de dominância, uma vez que queixadas possuem hierarquia social de dominância linear (NogUeIRA-FILHo et al., 1999; DuBost, 2001). Os resultados mostraram que a brincadeira, tanto social quanto solitária, no grupo de queixadas aumentou em relação ao controle, isso é, na ausência desses objetos. Observou-se ainda que os animais mais subordinados iniciavam as brincadeiras durante o período controle, mas após a introdução desses objetos, ocorreu o contrário, os dominantes iniciaram as brincadeiras com os subordinados. Este comportamento reflete aspectos da dominância dos animais dominantes sobre os subordinados, uma vez que os objetos novos podem ser considerados como "recurso limitado" os quais os dominantes detêm privilégios. 0 principal resultado deste estudo, no entanto, foi constatar que o uso de EA foi positivo para o bemestar dos animais, aumentando a brincadeira, diminuindo a inatividade e a agressividade de queixadas, além de validar o uso da brincadeira, um comportamento positivo, como um indicador de bem-estar para a espécie. Ao mesmo tempo, verificou-se que inatividade e agressividade podem ser consideradas como indicadores de bem-estar empobrecido (indicadores negativos) para a espécie (Nogueira et al., 2011b).
PAUL et al. (2005) apontaram a relação entre emoção e cognição como forma de compreender os estados afetivos em animais. O paradigma de viés de julgamento ou viés cognitivo busca analisar a interferência do estado emocional dos animais nos seus processos de tomada de decisão e, assim, pode fornecer informações importantes sobre o bem-estar psicológico dos animais e da valência positiva ou negativa deste estado (MENDL et al., 2009; MENDL et al., 2010; PAUL et al., 2005). Estudos sobre viés de julgamento (Doyle et al., 2010; Douglas et al., 2012) evidenciaram que os animais podem apresentar a capacidade de julgar um estímulo ambíguo como positivo ou negativo, dependendo de seu estado emocional (MENDL et al., 2009), podendo assim validar o bem-estar mental do indivíduo.

Buscando formas de mostrar o efeito de emoções negativas sobre a cognição de queixadas, Noguelra et al. (2015a) testaram o paradigma de viés cognitivo ou viés de julgamento para avaliar o impacto da caça na espécie, classificada como vulnerável pela IUCN (KeURoghlian et al., 2013). Queixadas são ameaçados tanto pela caça excessiva quanto pela destruição de seu habitat, uma vez que queixadas vivem em grupos de centenas de animais que precisam de áreas relativamente grandes para sobreviver (KEUROGHLIAN et al., 2013). Queixadas apresentam, como estratégia de defesa, o comportamento de ameaça, no qual o bando todo, sob a liderança dos animais mais dominantes, pode investir contra predadores, 
como os grandes felinos (SowLS, 1997). Há relatos de bandos de queixadas atacarem onça-pintada (Panthera onca, Linnaeus, 1758), um de seus principais predadores além do homem (SoWLS, 1997). Com a ampliação do uso de armas de fogo e com o conhecimento deste comportamento natural de queixadas, os caçadores passaram a abater um número cada vez maior de animais, começando por eliminar o líder, causando desorientação e desagregação do grupo como um todo. Não havia estudos sistemáticos que mostrassem o que ocorre no que diz respeito à tomada de decisão, com os animais do bando após um episódio de caça. Por este motivo, NogueIRA et al., (2015a) testaram o paradigma de viés cognitivo para avaliar o efeito da caça em queixadas. Para isso, os autores do estudo treinaram os animais por meio de condicionamento operante, a responderem sinais de comando para "ir" (apito, CS+) ou "não ir" (buzina, CS-) para o local de alimentação. Após os animais atingirem pelo menos $70 \%$ de acerto, os mesmos foram capturados com um puçá, instrumento usado rotineiramente para contenção, simulando um evento de caça. Em seguida, os animais foram desafiados a responder aos sinais de ir e não ir ao local de alimentação, como treinados anteriormente. No momento do teste, além dos sinais positivos e negativos, foi introduzido um sinal que não havia sido treinado anteriormente, o sinal ambíguo distinto dos sinais anteriores (som do sino de mesa, CSA). O teste de viés cognitivo é baseado na reação dos animais após a emissão do sinal ambíguo. Considera-se que, ao tomar a decisão de ir, o animal expressa um sinal otimista. Por sua vez, a decisão de não ir é considerada como uma visão pessimista em relação ao sinal ambíguo, mostrando, portanto, um viés de julgamento negativo em decorrência de seu estado emocional. O resultado deste estudo revelou que, após o evento de captura, queixadas se mostraram mais pessimistas, o que pode refletir em um estado emocional negativo, podendo fragilizar ainda mais os sobreviventes do bando, o que seria um dos fatores que tem contribuído para o declínio populacional da espécie (NogueIRA et al., 2015a).

Este paradigma tem sido usado com várias espécies e com objetivos diferentes, como por exemplo, analisar o bem-estar dos animais submetidos ao uso de gaiolas de metabolismo em estudos nutricionais (OLIVEIRA et al., 2016). A restrição de espaço devido ao uso dessas gaiolas pode gerar um estado emocional negativo nos animais e causar empobrecimento no seu bemestar. Nesse estudo, teve-se como objetivo testar o uso do paradigma de viés cognitivo/ julgamento acima descrito, para avaliar o efeito da restrição de espaço sob o estado emocional de caititus mantidos em baias de metabolismo em experimentos de nutrição. Seis animais foram submetidos a cinco testes de julgamento: em três dos testes os animais podiam se movimentar livremente, tanto na área de solário quanto nas baias de metabolismo (T1, T3 e T5), enquanto nos outros dois testes houve restrição maior de espaço, com os caititus presos nas baias de metabolismo (T2 e T4) (OLIVEIRA et al., 2016). Em T2 foi adicionado enriquecimento ambiental (EA), 
mas em T4 não houve a inclusão do EA. Antes de começar o estudo, os animais foram treinados por meio do condicionamento operante para "ir" ou "não ir" após a emissão de estímulos sonoros distintos. Ao atender ao comando "ir" (apito; CS+) o animal recebia uma recompensa (alimento) e durante o comando "não ir" (som de um caxixe; CS-) evitava a punição (jato d'água). Durante os testes de viés cognitivo, um terceiro sinal ambíguo (som de uma placa de alumínio; CSA) era apresentado. Após a emissão do sinal ambíguo, o animal não recebia a recompensa e tampouco recebia punição ao "ir" ou "não ir". Considerou-se como uma resposta otimista o "ir" após a emissão do sinal ambíguo e como resposta pessimista ao "não ir".

Adicionalmente, neste estudo, foram monitorados os metabólitos de glicocorticoides excretados nas fezes, para avaliar os níveis de estresse durante os tratamentos. O paradigma do viés cognitivo mostrou-se eficiente para analisar o efeito da redução de espaço sob o estado emocional dos caititus (OLIVEIRA et al., 2016). Durante os três primeiros testes (T1, T2 e T3) os caititus apresentaram proporções similares de respostas 'ir' ao CS +, CS- e CSA, mostrando-se, portanto, sem viés pessimista ou otimista. No teste de restrição maior de espaço em baias de metabolismo e sem enriquecimento ambiental (T4), os animais apresentaram viés de julgamento pessimista. No T4, a proporção de respostas "ir" após o estímulo ambíguo CSA foi similar à proporção verificada após a emissão do sinal negativo CS e ambas foram menores do que a proporção de respostas "ir" verificadas após o sinal positivo CS+. Adicionalmente, durante T1, T2 e T3, os caititus apresentaram níveis similares na concentração de metabólitos de glicocorticoides e inferiores aos níveis verificados em T4 e T5. Dessa forma, os efeitos negativos da restrição de espaço sem EA foram carreados para a fase controle seguinte, na qual os animais puderam movimentar-se livremente. Esse estudo pôde concluir que a restrição de espaço causa prejuízos ao estado afetivo dos animais e que, portanto, o bem-estar empobrecido apresentado pelos animais pode trazer um viés na interpretação dos resultados dos estudos nutricionais, uma vez que um estado emocional negativo pode interferir no consumo do alimento e, consequentemente, afetar os dados de digestibilidade dos nutrientes. Este efeito negativo, por outro lado, pode ser amenizado com a introdução de EA, que reduz o estresse dos animais causado pela restrição na movimentação quando contidos nas baias de metabolismo.

A bioacústica é outra linha de pesquisa que tem mostrado bons resultados na avaliação do bem-estar de animais (JON \& STOOKEY, 1999; GRADIM, 2001; MANTEUfFEl et al., 2004). O estado motivacional dos animais provoca alterações no batimento cardíaco e na movimentação da musculatura vocal, que em conjunto podem alterar a duração, a frequência mínima, a frequência máxima entre outros parâmetros acústicos de determinadas vocalizações e, por isso, podem ser selecionadas como indicadores de bem-estar (BRIEFER, 2012). Nesta linha de 
estudo, NogueIRA et al. (2016) avaliaram o efeito do uso da técnica de EA alimentar sobre as vocalizações de queixadas, como também monitoraram a excreção de metabólitos de glicocorticoides para avaliar o nível de estresse nos animais. Primeiro, os autores descreveram os parâmetros acústicos das vocalizações, tanto nas fases controle do estudo (sem EA), quanto nas fases de tratamento (com EA). Encontraram três tipos de vocalizações frequentes que foram o bater de dentes, o grunhido e o latido. Como resultado das análises dos parâmetros acústicos, verificaram-se diferenças nas frequências máximas das vocalizações batida de dentes e grunhido entre as fases do estudo (com EA e sem EA). Ambas as vocalizações ocorrem durante a disputa por alimento. Nas fases com EA, a frequência máxima destas vocalizações foi mais baixa do que durante as fases controle sem EA, revelando que há um estado motivacional diferente dependendo do ambiente em que o animal está exposto (NogueIRA et al., 2016).

Apesar da indicação de diferenças no estado afetivo dos animais, não foram detectadas diferenças nas concentrações de metabolitos de glicocorticoides nas fezes dos animais coletadas nas diferentes fases experimentais. NoguEIRA et al. (2016) sugerem que este resultado seria explicado pelas condições gerais do ambiente em que estes animais estavam sendo criados: sistema semi-confinado em piquetes construídos em área florestada. Os resultados mostraram, portanto, que o espaço disponível foi adequado para os animais e que as boas condições gerais propiciaram conforto, que se refletiu em baixo nível de estresse, verificado pelas medidas endócrinas. Por outro lado, o monitoramento dos parâmetros acústicos permitiu a detecção de características mais sutis, possibilitando avaliar o efeito positivo do EA proposto. Esse estudo mostra, portanto, que medidas de monitoramento acústico podem ser uma ferramenta útil para avaliar o estado de bemestar de queixadas e deve ser testado em outras espécies.

\section{Lacunas no Conhecimento e Perspectivas Futuras SOBRe BEM-ESTAR de MAMífEROS NEOTROPICAIS}

Tanto a ausência de ambientes complexos no cativeiro quanto o manejo inadequado de mamíferos neotropicais podem impactar enormemente o seu desenvolvimento psíquico e fisiológico, como já descrito neste manuscrito e também observado para outras espécies mantidas em zoológicos (LIU et al, 2003; MASON \& LATHAN, 2004; HOSEY, 2005). Alguns estudos mostraram que para mamíferos neotropicais, tanto do ponto de vista de bem-estar animal quanto econômico, deve ser adotado o sistema de produção em semi-confinamento (ANDRADE et al., 2001; Nogueira-Filho \& Nogueira, 2004; Nogueira-FilHo et al., 2004; ANDRADE et al., 2009; NogueIRA-FILHo et al., 2013). Sob estas condições, não há relatos da ocorrência de comportamentos anormais que impactem a saúde dos animais ou a produção dos mesmos. No entanto, como relatado anteriormente sobre o uso de enriquecimento ambiental (EA) em 
queixadas (NogueIRA et al., 2011b), os resultados obtidos são claros sobre os benefícios de objetos novos com os quais os animais interagem positivamente e aumentam comportamentos tais como a brincadeira e diminuem a agressividade. É necessário, portanto, que novos estudos avaliem o uso de enriquecimento ambiental para outras espécies de animais neotropicais mantidos em diferentes sistemas de produção, para avaliar seu efeito na diminuição da previsibilidade do ambiente de cativeiro e melhorias do bem-estar desses animais. É preciso também criar novos métodos para o fornecimento de alimento, como o uso de comedouros desafio (NogueIRA et al., 2011a), que estimulam os caititus a buscar/ trabalhar para ter acesso ao alimento e, com isso, mantem os animais ativos e motivados. Observase, no entanto, que existe pouca preocupação na inovação do ambiente e manejo alimentar, por parte de zoológicos e centros de conservação que mantem pecaris, como caititus e queixadas, por exemplo. Estes recintos em geral, não têm complexidade, mostrando pouco cuidado com o bem-estar dos animais e consequentemente poucos atrativos aos visitantes.

Em um estudo sobre a avaliação do temperamento de caititus e queixadas (NogUEIRA et al. 2015b), foram observadas diferenças nos traços comportamentais entre os indivíduos de ambas as espécies. De forma geral, apesar de queixadas terem sido considerados mais reativos do que os caititus, observaram-se variações nos tipos de temperamento (personalidade) dentro de cada uma das espécies. Estudos desse tipo, portanto, podem ser usados para subsidiar um programa de seleção de animais mais mansos e que melhor se adaptem ao cativeiro e, consequentemente, trazer melhorias ao bemestar destes animais, com menos sofrimento devido ao manejo humano. Nogueira et al. (2004), verificaram que mesmo a capivara, geralmente considerada dócil e relativamente calma, sofre com a presença humana. Nesse estudo, os autores mostraram que capivaras adultas, capturadas na natureza e há dois anos mantidas em cativeiro, mostravam o efeito do estresse causado pelo manejo diário de limpeza das baias e permaneciam mais tempo no tanque d'água durante a presença do tratador no recinto do que capivaras nascidas em cativeiro (NogUEIRA et al., 2004).

\section{CONSIDERAÇÕES FINAIS}

Nesta revisão foram descritos os desafios que animais neotropicais enfrentam ao serem mantidos em cativeiro. Foi mostrado que, apesar dos animais não sofrerem o estresse da falta e busca constante por alimento, que ocorre no ambiente natural, a monotonia do cativeiro pode afetar seu bem-estar. Para contrapor, foram mostrados alguns exemplos de técnicas para detectar problemas e promover o bem-estar de mamíferos neotropicais. Também foi realçado que aspectos do comportamento social devem ser respeitados para que se alcance sucesso em sua produção de mamíferos neotropicais. Por outro lado, foi mostrado que a plasticidade comportamental, exibida por algumas espécies, pode ser aproveitada para estabelecer práticas 
de manejo que viabilizem a atividade, sem comprometer o bem-estar animal. Finalmente, foram apontadas as lacunas de conhecimento, especialmente os relativos aos comportamentos típicos e/ou anormais das espécies neotropicais. É importante, portanto, incentivar a realização de mais pesquisas que visem a descrição do repertório comportamental dessas espécies, seja em ambiente natural ou em cativeiro, para que se possa reconhecer e classificar seus padrões comportamentais e, com isso, estabelecer praticas que promovam melhorias em seu bem-estar. Finalmente, recomenda-se que as técnicas aqui apresentadas e discutidas sejam aperfeiçoadas e testadas na produção de animais neotropicais mantidos em cativeiro com diferentes objetivos, como zoológicos, centros de triagem e reabilitação, centros de multiplicação de animais destinados a programa de soltura e especialmente nas criações comerciais.

\section{REFERÊNCIAS BIBLIOGRÁFICAS}

Abreu Bovo, A.A.; FERRAZ, K.M.P.M.B.; MoreiRA, J.R.; GHeler-COSTA, C.; LYRAJORGE, M.C.; VERDADE, L.M. 2016. Capybaras (Hydrochoerus hydrochaeris) in anthropogenic environments: challenges and conflicts, pp. 178-189. In: GhelerCosta, C.; Lyra-Jorge, M.C.; Verdade, L.M. (eds.). Biodiversity in agricultural landscapes of Southeastern Brazil. Berlin: De Gruyter Open. 334 p.

ANdRADE, P.C.M.; Costa, P.M.; CANTO, S.L.O.; DUARTE, J.A.M. \& SILVA F.F. 2001. Manejo semi-intensivo de capivaras (Hydrochoerus hydrochaeris) como alternativa de produção para pequenos produtores nas várzeas amazônicas. In: IV Encontro da Sociedade Brasileira de Sistemas de Produção, Belém, Brasil. pp. 1-15.

ANDRADE, P.C.M.;AlveS DASILVA, R.J., MOTADUARTE, J.A., CANTO, S.L.O.; COSTA, P.M.; Oliveira, P.H.G., MonteIRO, M.S., BOMFIM, S.V.A., Oliveira-Pereira, M.I., Pereira-Filho, M., Oliveir, A.B., Rodrigues, W.S., SOUSA, A.O. 2009 Manejo e alimentação de caitetus (Tayassu tajacu) e queixadas (T. pecari) em cativeiro na Amazônia Central. Relatório Técnico. Disponível em < https://www. researchgate.net/publication/319003651_ Manejo_e_Alimentacao_de_Caitetus_ Tayassu_tajacu_e_Queixadas_T_pecari_ em_cativeiro_na_Amazonia_Central>. Acesso em: 5 out 2017.

BECK, H. 2005. Seed predation and dispersal by peccaries throughout the Neotropics and its consequences: a review and synthesis, pp. 77-115. In: FORGET, P.M.; LAMBERT, J.E.; HULME, P.E. \& VANDER WALL, S.B. (eds). Seed fate: predation, dispersal and seedling establishment. Cambridge, CABI. 400 p.

BEKOFF, M. \& BYERS, J.A. 1998. Animal play: Evolutionary, comparative and ecological approaches. Nova York, Cambridge University Press. 262 p.

Barros, K.S.; TOKUmaru, R.S.; Pedrosa, J.P. \& 
NogueIRA, S.S.C. 2011. Vocal repertoire of captive capybara (Hydrochoerus hydrochaeris): structure, context and function. Ethology 116: 1-11.

BARBOZA, R.D.; LOPES, S.F.; SOUTO, W.M.S.; FERnANDES-FERREIRA, H. \& Alves, R.R.N. 2016. The role of game mammals as bushmeat in the Caatinga, northeast Brazil. Ecology and Society 21 (2): 2.

BeCK-KIng, H.; Helversen, O.; BeCK-KIng, R. 1999. Home range, population density, and food resources of Agouti paca (Rodentia: Agoutidae) in Costa Rica: A study using alternative methods. Biotropica 31: 675685.

BODMER, R.E. \& RoBINSON, J.G. 2004. Evaluating the sustainability of hunting in the Neotropics, pp. 299-323. In: SILVIUS, K.; Bodmer, R. \& Fragoso, J. (eds.). People in nature, wildlife conservation in South and Central America. Nova York, Columbia University Press. 464.

Bodmer, R.E., Pezo Lozano, E. \& FAng, T.G. 2004. Economic analysis of wildlife use in the Peruvian Amazon, pp. 191-207. In: SILVIUS, K.; Bodmer, R. \& Fragoso, J. (eds.). People in nature, wildlife conservation in South and Central America. Nova York, Columbia University Press. 464.

BRIEFER, E.F. 2012. Vocal expression of emotions in mammals: mechanisms of production and evidence. Journal of Zoology 288:
$1-20$.

Bulte, E.H. \& DamaniA. R. 2005. An economic assessment of wildlife farming and conservation. Conservation Biology 19 (4):1222-1233.

CAWTHORN, D.M. \& HOFFMAN L.C. 2016. Controversial cuisine: A global account of the demand, supply and acceptance of "unconventional" and "exotic" meats. Meat Science 120: 19-36.

ChaucA, L. 1997. Producción de cuyes (Cavia porcellus). Roma, FAO. $78 \mathrm{p}$.

Douglas, C.; Bateson, M., Walsh, C., Bedue, A. \& EDWARDS, S. 2012. Environmental enrichment induces optimistic cognitive biases in pigs. Applied Animal Behaviour Science 139: 65-73.

DOYLE, R.E.; VIDAL, S.; HINCH, G.N.; FISHER, A.D.; BoISSY, A. \& LEE, C. 2010. The effect of repeated testing on judgement biases in sheep. Behavioural Processes 83: 349352.

Dubost, G. 2001. Comparison of the social behaviour of captive sympatric peccary species (genus Tayassu): correlation with their ecological characteristics. Mammalian Biology 66: 65-83.

FAgEN, R. 1981. Animal Play Behavior. Oxford: Oxford University Press. 684 p.

FERRAZ, K.M.P.M.B.; IZAR, P.; SATO, T.; NISHIDA S.M. 
2013. Social and spatial relationships of capybaras in a semi-con fined production system, pp 243-260. In: Moreira, J.R.; FERRAZ, K.M.P.M.B.; HeRRERA, E.A. \& MACDONALD, D.W. (eds). Capybara: Biology, use and conservation of an exceptional Neotropical species. Londres, SpringerVerlag. 408 p.

Figueira, M.L.O.A.; Carrer, C.R.O. \& Silva Neto, P.B. 2003. Ganho de peso e evolução do rebanho de queixadas selvagens em sistema de criação semi-extensivo, em reserva de cerrado. Revista Brasileira de Zootecnia 32: 191-199.

Figueroa-de león, A.; Naranjo, E.J.; Perales, H.; SAntos-Moreno, A. \& LoRenzo C. 2016. Availability and characterization of cavities used by pacas (Cuniculus paca) in the Lacandon Rainforest, Chiapas, Mexico. Revista Mexicana de Biodiversidad 87: 1062-1068.

FRAGOSO, J.M.V. 1998. Home range and movement patterns of white-lipped peccary (Tayassu pecari) herds in the northern Brazilian Amazon. Biotropica 30: 458-469.

Gongora, J., Reyna-Hurtado, R., BeCK, H., Taber, A., Altrichter, M. \& Keuroghlian, A. 2011. Pecari tajacu. The IUCN Red List of Threatened Species 2011. Disponível em: <http://dx.doi.org/10.2305/IUCN. UK.2011-2.RLTS.T41777A10562361.en> Accesso em 8 out. 2017
GRANDIM, T. 2001 Cattle vocalizations are associated with handling and equipment problems at beef slaughter plants. Applied Animal Behaviour Science 71: 191-201.

HeffineR, C.L. 2004. Research methods for education, psychology and the social sciences. Disponível em: <http://www. allpsych.com/researchmethods $>$. Accesso em 8 out. 2017.

HeLD, A.D.E. \& SpINKA, M. 2011. Animal play and animal welfare. Animal Behaviour 81: 891899.

HerRerA, E.A. 2013. Capybara social behavior and use of space: patterns and processes, pp 195-207. In: MoreIRA, J.R.; FERRAZ, K.M.P.M.B.; HERRERA, E.A. \& MACDONALD, D.W. (eds). Capybara: Biology, use and conservation of an exceptional neotropical species. Londres, Springer-Verlag. 408 p.

HofFMAN, L.C. 2016. Game as an alternative protein source: feed science. AFMA Matrix 25 (4): 54-55.

Hosey, G. 2005. How does the zoo environment affect the behaviour of captive primates? Applied Animal Behaviour Science 90: 107-129

Hosey, G.; Melfi, V. \& PANkhurst, S. 2009. Zoo animals - behaviour, management, and welfare. Oxford, Oxford University Press. $613 \mathrm{p}$

ISAAC, V.J.; AlmeIDA, M.C.; GIARRIZZO, T.; DeUS, 
C.P.; Vale, R.; Klein, G.; Begossi, A. 2015. Food consumption as an indicator of the conservation of natural resources in riverine communities of the Brazilian Amazon. Anais da Academia Brasileira de Ciências 87 (4): 2229-2242.

JON, M.W. \& STOOKEY, M.J. 1999. Effects of restraint and branding on rates and acoustic parameters of vocalization in beef cattle. Applied Animal Behaviour Science 62: 125-135.

Keuroghlian, A.; Desbiez, A.; Reyna-Hurtado, R.; Altrichter, M.; Beck, H.; TABer, A. \& Fragoso, J.M.V. 2013. Tayassu pecari. In: IUCN 2013. IUCN Red List of Threatened Species. Version 2013.1. Disponível em: $<\boldsymbol{w w w}$.iucnredlist.org >. Accesso em 8 out. 2017.

KYLE, R. 1994. New species for meat production. The Journal of Agricultural Science 123: $1-8$.

Lacerda, P. O.; TOKumaru, R. S. \& Nogueira, S.S.C. 2013. Vocal signature in capybara, Hydrochoerus hydrochaeris. Acta Ethologica 45:12-19.

Le Pendu, Y.; Guimaraes, D.A.; Linhares, A. 2011. Estado da arte sobre a criação comercial da fauna silvestre brasileira. Revista Brasileira de Zootecnia 40: 52-59.

LIU, D.; Wang, Z.; TIAN, H.; YU, C.; ZHANG, G.; WEI, R. \& ZHANG, H. 2003. Behavior of giant pandas (Ailuropoda melanoleuca) in captive conditions: Gender differences and enclosure effects. Zoo Biology. 22: 77-82.

LobÃo, E. S. P. \& Nogueira-Filho, S.L.G. 2011. Human-wildlife Conflicts in the Brazilian Atlantic Forest. Suiform Soundings 10: 1422.

MACDONALD, D.W.; KIANTZ, K. \& APLIN, R.T.1984. Behavioural, anatomical and chemical aspects of scent marking amongst capybaras (Hydrochoerus hydrochaeris) (Rodentia: Caviomorpha). Journal of Zoology 202: 341-360.

Manteuffel, G.; Puppe, B. \& SChön, P.C. 2004. Vocalization of farm animals as a measure of welfare. Applied Animal Behaviour Science 88: 163-182.

MASON, G.J. \& LATHAM, N.R. 2004. Can't stop, won't stop: is stereotypy a reliable animal welfare indicator. Animal Welfare 13: 5769.

Mason, G; R. Clubb, R; Latham, N. \& Vickery, S. 2007. Why and how should we use environmental enrichment to tackle stereotypic behaviour? Applied Animal Behaviour Science 102: 163-188.

MATTOS, A.A. 2015. Uso de alimentos alternativos na criação da paca (Cuniculus paca). Dissertação de Mestrado.Universidade Estadual de Santa Cruz. 98 p.

Mendes, A. \& Nogueira-Filho, S.L.G. 2013. Feeds 
and nutrition of farmed capybaras, pp. 229-241. In: MoReIRA, J.R.; FERRAZ, K.M.P.M.B.; Herrera, E.A. \& Macdonald, D.W. (eds). Capybara: Biology, use and conservation of an exceptional neotropical species. Londres, Springer-Verlag. 408 p.

MendL, M.; Burman, O.H.P. \& PAUL, E.S. 2009. Cognitive bias as an indicator of animal emotion and welfare: Emerging evidence and underlying mechanisms. Applied Animal Behaviour Science 118: 161-81.

MendL, M.; Burman, O.H.P. \& PAUL, E.S. 2010. An integrative and functional framework for the study of animal emotion and mood. Proceedings of the Royal Society B: Biological Sciences 277: 2895-2904.

Metre, T.K.; TSONGO, F.; Mugisho, A.B.; KAMPEMBA, F.M.; AYAGIRWE, R.B.B.; AZINE, P.C. \& CHIURI, W.L. 2014. From taboo to commodity: History and current situation of cavy culture in the Democratic Republic of the Congo. Livestock Research for Rural Development Volume 26, Artigo 151 Disponível em: <http://www.Irrd.org/ Irrd26/8/maas26151.htm>. Accesso em 10 out. 2017.

MCPheE, M.E. \& CARLSteAd, K. 2012. The importance of maintaining natural behaviors in captive mammals, pp. 303313. In: Devra G. Kleiman, Katerina V. THOMPSON, AND CHARLOTTE KIRK BAER (eds.). Wild mammals in captivity: Principles and techniques for zoo management. 2 ed. Chicago, University of Chicago Press. 592 p.

Monego, E.T.; Peixoto, M.R.G. \& Cordeiro, M.M. 2010. Food insecurity of Tocantins's quilombolas communities. Segurança Alimentar e Nutricional 17: 37-47.

MONTEIRO, M.S. 2014.Coeficientesdedigestibilidade de nutrientes de alimentos usados na criação de paca (Cuniculus paca). Dissertação de Mestrado.Universidade Estadual de Santa Cruz. $85 \mathrm{p}$.

Morgan, K.N. \& TROMBorg, C.T. 2007. Sources of stress in captivity. Applied Animal Behaviour Science 102: 262-302.

NOGUEIRA-NETO, P. 1973. Criação de animais indígenas vertebrados: Peixes, anfíbios, répteis, aves e mamíferos. São Paulo, Edições Tecnapis. 327 p.

Nogueira, S.S.C.; NogueIRA-FILHO, S.L.G.; OtTA, E.; Dias, C.T.S. \& Carvalho, A. 1999. Determination of the causes of infanticide in capybara (Hydrochaeris hydrochaeris) groups in captivity. Applied Animal Behaviour Science 62: 351-357.

NOGUeIRA, S.S.C.; OTTA, E.; DIAS, C.T.S. \& NOGUEIRA FILHO, S.L.G. 2000. Alloparental behaviour in capybara (Hydrochoerus hydrochaeris). Revista de Etologia 2 (1): 17-21.

Nogueira, S.S.C.; BernaRdI, L.G. \& NogUeIRAFILHO, S.L.G. 2004. A note on comparative enclosure facilities used by wild and 
captive born capybara (Hydrochoerus hydrochaeris). Applied Animal Behaviour Science 89:139-143.

NogueIRA, S.S.C; SILVA, M.G.; DIAS, C. T. S.; Pompéia, S.; Cetra, M. \& Nogueira-Filho, S. L. G. 2010. Social behaviour of collared peccaries (Pecari tajacu) under three space allowances. Animal Welfare 19: 243-248.

Nogueira, S.S.C. \& NogueIRA-Filho, S.L.G. 2011. Wildlife farming: an alternative to unsustainable hunting and deforestation in Neotropical forests? Biodiversity and Conservation 20: 1385-1397.

Nogueira, S.S.C.; Calasans, S.G.; Costa T.S.O.; Peregrino, H. \& Nogueira-Filho, S.L.G. 2011a. Effects of varying feed provision on behavioral patterns of farmed collared peccary (Mammalia, Tayassuidae). Applied Animal Behaviour Science 132:193-199.

Nogueira, S. S. C.; Soledade, J. P.; PompéiA, S. \& NogueirA-Filho, S.L.G. 2011b. The effect of environmental enrichment on play behaviour in white-lipped peccary (Tayassu pecari). Animal Welfare 20: 505.

NOGUEIRA, S.S.C. \& NOGUEIRA-FILHO, S.L.G. 2012. Capybara (Hydrochoerus hydrochaeris) behaviour and welfare: implications for successful farming practices. Animal Welfare 21: 527-533.

Nogueira, S.S.C.; Abreu, S. A.; Peregrino, H. \& NogUeIRA-FILHO, S. L. G. 2014. The effects of feeding unpredictability and classical conditioning on pre-release training of white-lipped peccary (Mammalia, Tayassuidae). PlosOne 9: 1-6.

Nogueira S.S.C., Fernandes I.K., Costa T.S.O., NogUeIRA- FILHo S.L.G. \& MeNDL M., 2015a. Does trapping influence decision-making under ambiguity in white-lipped peccary (Tayassu pecari)? PloS One 10: 1-12.

Nogueira, S.S.C.; Macedo, J.; SAnt'Anna, A. \& NogUeIRA-FILHo, S.L.G. 2015b. Assessment of temperament traits of white-lipped (Tayassu pecari) and collared peccaries (Pecari tajacu) during handling in a farmed environment. Animal Welfare 24: 291-298.

Nogueira, S.S.C.; Lacerda, Z.; MAgalhães, M. ; Pereira Neto, J.S.; Abreu, S.A.; Silva, H.P.A.; NogUeIRA-FILHO, S.L.G. \& LIMA, S,G. C. 2016. The use of white-lipped peccary (Tayassu pecari) vocalizations as welfare indicator. Suiform Soundings 15 (1): 77-85.

NogueIRA-FILHO, S.L.G. 1999. A criação de cateto e queixada. Viçosa, Centro de Produções Técnicas. $50 \mathrm{p}$.

NogueirA-Filho, S.L.G. \& NogUeIRA, S.S.C. 1999. Criação de pacas (Agouti paca). Piracicaba, FEALQ. 60 p.

Nogueira-Filho, S.L.G.; NogUeira, S.S.C. \& SATO, T. 1999. A estrutura social de pecaris (Mammalia, Tayassuidae) em cativeiro. Revista de Etologia 1 (2): 89-98. 
Nogueira-Filho, S.L.G.; NogueIRA, S.S.C.; MENDES, A. \& JORI, F. 2004. The commercial production of collared peccary (Tayassu tajacu) an example of wildlife ranching from Bahia, Brazil. In: 6th International WIldlife Ranching Symposium, Paris, França. pp. 83-84.

NogueirA-Filho, S.L.G. \& Nogueira, S.S.C. 2004. Captive breeding programs as na alternative for wildlife conservation in Brazil, pp 171-190. In: SILVIUS, K.; BOdMER, R. \& Fragoso, J. (eds.). People in nature, wildlife conservation in South and Central America. Nova York, Columbia University Press. 464.

Nogueira-Filho, S.L.G. \& Nogueira, S.S.C. 2013. The impact of management practices on female capybara reproductive parameters in captivity, pp. 180-185. In: MOREIRA, J.R.; FerRaz, K.M.P.M.B.; HerRera, E.A. \& MACDONALD, D.W. (eds). Capybara: Biology, use and conservation of an exceptional Neotropical species. Londres, SpringerVerlag. $408 \mathrm{p}$.

Nogueira-Filho, S.L.G.; Pinheiro, M.S. \& NogueIRA, S.S.C. 2013. Confined and semi-confined production systems for capybaras, pp. 146-149. In: MOREIRA, J.R.; FerRaz, K.M.P.M.B.; Herrera, E.A. \& MACDONALD, D.W. (eds). Capybara: Biology, use and conservation of an exceptional neotropical species. Londres, SpringerVerlag. $408 p$.
Nogueira-Filho, S.L.G.; Bastos, I.H.; Mendes A. \& Nogueira, S.S.C., 2016. Protein requirements of finishing paca (Cuniculus paca). Tropical Animal Health and Production 48: 1005-1011.

Nogueira-Filho, S.L.G.; LOPES, P.C.; Ferreira, D. \& NogUeIRA, S.S.C. 2017. Flexibility in the social behavior of captive female capybaras (Mammalia, Rodentia). Behavioural Processes, 142: 29-32.

Oliveira, F.R.M.; NogueIRA-FILHO, S.L.G.; SOUSA, M.B.C.; DiAS, C.T.S. MendL, M. \& NogUeIRA, S.S.C. 2016. Measurement of cognitive bias and cortisol levels to evaluate theeffects of space restriction on captive collared peccary (Mammalia, Tayassuidae) Applied Animal Behaviour Science 181:76-82.

Paul, e.S.; Harding, e.J. \& Mendl, M. 2005. Measuring emotional processes in animals: the utility of a cognitive approach. Neuroscience and Biobehavioral Reviews 29: 469-492.

Pitman R T., Fattebert J., Williams S. T, Williams K. S, HiLl R A, HUNTER L. T B, SLOtOW R. \& BALME, G.A. 2017. The conservation costs of game ranching. Conservation Letters 10 (4): 403-413.

REDFORD, K.H. 1992. The empty forest. Bioscience 42: 412-422.

Sabatini, V. \& Paranhos Da Costa, M.J.R. 2001. Caecotrophy in pacas (Agouti paca, 
Linnaeus, 1766), Mammalian Biology66: 305-307.

Silva Neto, B.C.; Nascimento, A.L.B.; Schiel, N.; Alves, R.R.N.; Souto, A. \& Albuquerque, U.P. 2016. Assessment of the hunting of mammals using local ecological knowledge: an example from the Brazilian semiarid region. Environment, Development and Sustainability 19 (5): 1795-1813.

SMYTHE, N. 1991. Steps toward domesticating the paca (Agouti=Cuniculus paca) and prospects for the future, pp. 202-216. In: Robinson, J.G. \& Redford, K.H. (eds.). Neotropical Wildlife use and Conservation. Chicago, University of Chicago Press. 538 p.

SoWLS, L.K. 1997. Javelinas and other peccaries: their biology, management, and use. 2 ed. Tucson, University of Arizona Press. 354 p.

STAHL, P.W. 2003. Pre-Columbian Andean animal domesticates at the edge of empire. World Archaeology 34: 470-483.

Van Vliet, N.; Cruz, D., Quiceno-Mesa, M.; NeVes de Aquino, L.; Moreno, J.; RAIRon, R. \& FA, J. 2015. Ride, shoot, and call: wildlife use among contemporary urban hunters in Três Fronteiras, Brazilian Amazon. Ecology and Society 20 (3): 8.
VAn Vliet, N.; Cornelis, D.; Beck, H.; Lindsey, P.; NASI, R.; LebeCK, S.; MOREnO, J.; Fragoso, J. \& JORI, F. 2016. Meat from the wild: extractive uses of wildlife and alternatives for sustainability. In: MATEO, R.; ARROYO, B. \& GarcíA, J.T. (eds.). Current trends in wildlife research. Suiça, Springer. 293 p.

WAITT, C. \& BUCHANAN-SMITH, H.M. 2001. What time is feeding? How delays and anticipation of feeding schedules affect stump-tailed macaque behavior. Applied Animal Behaviour Science 75: 75-85.

WATERS, J.V. 2009. Toward a predictive theory for environmental enrichment. Zoo Biology 28: 609-622.

Zeder M. A. 2012. Pathways to animal domestication, pp. 227-259. In: GEPTS, P.; FAmula, T.R. \& Bettinger, R.L. (eds.). Biodiversity in agriculture: domestication, evolution, and sustainability. Cambridge, Cambridge University Press. 630 p.

Recebido: 25/10/2017

Revisado: 28/12/2017

Aceito: 10/01/2018 